\title{
Les contradictions du génome
}

Une routine abrutissante?

C'est bien ainsi que sont souvent perçus les "programmes Génome" développés dans plusieurs pays. Beaucoup imaginent qu'ils reposent, dans des laboratoires dédiés à cet usage, sur une armée de techniciens effectuant des tâches répétitives et sans imagination sous la houlette de quelques chercheurs. Selon cette vision assez répandue il ne s'agit en somme que de répéter, presque à l'infini, les gestes courants du laboratoire de biologie moléculaire ; l'on imagine certes que quelques opérations sont prises en charge par des automates, mais l'ensemble du processus apparaît singulièrement peu créatif.

Pour ceux qui connaissent réellement les laboratoires impliqués il est clair que cette vision est dans une très large mesure fausse. Le génie génétique n'a qu'une quinzaine d'années d'existence, et il est encore en évolution rapide. Les méthodes bougent, progressivement ou par à-coups (que l'on songe à ce qu'ont changé la PCR, ou la mise au point des YAC...) et la stratégie optimale à un moment donné peut devenir très vite caduque. C'est d'ailleurs ce qui explique en grande partie la faible automatisation des laboratoires, car la mise au point d'appareils efficaces demande du temps et il est difficile de l'effectuer dans un contexte aussi changeant. Cette évolution perpétuelle a deux conséquences : la nécessité de changer son fusil d'épaule à intervalles rapprochés, afin de ne pas poursuivre une opération selon une technique maintenant obsolète et remplaçable par une autre cinq ou dix fois plus efficace; et, d'autre part, le fait que les méthodes n'ont routine bien au point. Il faut donc que le personnel soit capable d'apprécier l'importance de nouveautés, de les mettre en œuvre, et également de comprendre ce qui se passe quand l'expérience ne donne pas les résultats attendus : ce que nos collègues anglo-saxons désignent du nom très expressif de trouble-shooting.

Il reste néanmoins vrai que l'affinement de la carte génétique humaine, la construction de contigs de clones s'étendant sur des dizaines de mégabases et, bien sûr, le séquençage de grandes régions d'ADN sont des entreprises comportant une part non négligeable d'un travail répétitif dont l'exécution doit être impeccable sous peine d'arriver à un rapport qualité/prix désastreux : pas question ici de tolérer l'improvisation un peu brouillonne qui est souvent rencontrée dans nos laboratoires " académiques ". Un tel manque d'organisation est admissible à la rigueur pour des travaux de petite ampleur, dans un cadre académique et au cours d'une phase de découverte de la recherche par des étudiants. Mais elle n'a pas sa place dans de grands programmes mettant en jeu des appareillages relativement sophistiqués et - du fait même de cette automatisation - des quantités considérables de réactifs coûteux : pensons à la consommation de l'enzyme Taq polymérase dans un centre comme celui de Maynard Olson et David Schlessinger à SaintLouis (MO, USA) qui effectue près de mille réactions de PCR par jour...

\section{Comment s'organiser?}

Les solutions apportées sont très variables et j'en ai observé diverses variétés au cours de mes visites de laboratoires à travers le monde. Certains pratiquent le " tout technicien ", c'est-à-dire ont recours à du personnel d'exécution, formé à une tâche précise et pouvant l'effectuer - on l'espère - avec fiabilité et sans trop de lassitude pendant plusieurs années. C'est par exemple le cas du laboratoire de Tada-Aki Hori au Japon, qui assure la localisation par hybridation in situ des très nombreux cosmides isolés par l'excellent groupe de Yasuke Nakamura (Tokyo) à partir des chromosomes 3 et 11 . L'équipe de Hori, installée dans un centre de recherches de la Science and Technology Agency (un peu l'équivalent de notre CEA et du Department of Energy américain) à Chiba, près de Tokyo, pratique l'in situ non radioactif avec un matériel classique. Les microscopes sont équipés d'appareils photo ; pas de caméra CCD et encore moins de microscope confocal. Les localisations reposent sur le travail de trois techniciennes, supervisées par un chercheur, qui effectuent plusieurs centaines de localisations par an. Les résultats sont de bonne qualité, et les opératrices paraissent contentes de leur sort. Le Généthon, dont nous reparlerons plus loin, a fait lui aussi le choix d'employer essentiellement du personnel technique, encore qu'il ait été amené à revoir à la hausse le niveau de formation demandé après quelques déboires initiaux; enfin certains Genomes centers aux États-Unis comme celui que dirige Rick Myers à San Francisco (chromosome 4) ou Glen Evans au Salk Institute (chromosome 11) fonctionnent de la même façon. Il faut noter à cet égard que le terme de technicien recouvre aux États-Unis une réalité assez différente 
de ce qu'elle est en France: dans cette société très mobile beaucoup sont en fait des étudiants qui jouent ce rôle pendant deux ou trois ans, le temps de faire des économies avant de reprendre leurs études et d'achever leur maîtrise ou de commencer un doctorat. Il y a donc dans cette catégorie un renouvellement constant et une motivation que la routine n'a pas eu le temps d'émousser; quant aux difficultés de positionnement que nous connaissons souvent avec nos techniciens professionnels, elles se posent autrement dans ce contexte.

D'autres laboratoires choisissent au contraire d'employer surtout des étudiants et postdoctorants. C'est alors que le caractère systématique du travail commence à poser problème. $\mathrm{Si}$ la tâche qui consiste à établir une carte physique complète du chromosome 7, par exemple, a une justification scientifique indéniable, elle n'en comporte pas moins une part importante de travail répétitif. C'est le cas de toute recherche en génie génétique, où l'on finit toujours par passer beaucoup de temps à préparer des ADN, effectuer des hybridations, des réactions PCR, couler, faire migrer et interpréter des gels... mais la " rentabilité " de tels exercices semble beaucoup plus élevée lorque l'on travaille sur une maladie génétique que dans le cadre d'une cartographie systématique. Le post-doc qui trime sur la recherche de gènes contenus dans un jeu de cosmides provenant du bras court du chromosome 4 peut toujours rêver qu'il va découvrir le gène de la maladie de Huntington, et qu'il aura les honneurs de revues comme Cell, Science ou Nature. En tout état de cause, pourvu qu'il isole quelques YAC, détermine leur carte et y repère grâce aux îlots $\mathrm{CpG}$, aux $Z o o$ blots ou à toute autre méthode quelques gènes, qu'il montre dans quel tissu ces derniers sont transcrits... il aura assemblé les éléments d'un article honorable qu'il pourra soumettre à Genomics ou à Nucleic Acids Research. Il tirera aussi de ce travail les éléments de quelques séminaires et communications dans les colloques. Il aura enfin le sentiment de maîtriser, en quelque sorte d'être propriétaire, de ces résultats et d'avoir apporté à l'édifice de la biologie une pierre - petite sans doute mais bien identifiable et résultant indéniablement de sa propre activité.

Pourtant on ne peut pas dire qu'un tel travail soit réellement créatif, ni au niveau conceptuel ni au niveau technique : il peut se limiter à l'application de méthodes éprouvées sans idée $a$ priori et sans que l'inventivité n'entre en jeu. Ce sera pourtant une recherche qualifiée de fondamentale, ce qu'elle est peut-être dans son objet mais, à mon avis, pas dans sa démarche. La contribution ainsi apportée est limitée, entre autres, par le fait qu'elle ne s'intègre pas facilement dans une vision d'ensemble, que le degré de précision et de validité des informations obtenues n'a pas été soumis à une évaluation dont les critères soient bien définis, et que seules les données présentées dans une éventuelle publication - qui sera sans doute très abrégée vu son intérêt limité - finiront éventuellement par être consignées dans les banques de données. On peut facilement argumenter que, sauf exception, les efforts ainsi investis seront moins utiles à l'avancement des connaissances que le travail qu'aurait mené le même post-doc dans le cadre d'une entreprise génomique de grande ampleur comme celle qui est développée par Maynard Olson et David Schlessinger. Pourtant dans le deuxième cas le mieux qu'il puisse espérer est d'être signataire, avec beaucoup d'autres, d'un ou deux articles relatant quelques étapes de l'avancement de la carte d'un chromosome.

Une solution partielle consiste à organiser le travail afin que chaque chercheur ait une double activité : d'une part une participation à un projet général, peu productif dans l'immédiat mais constituant l'ossature de la stratégie du laboratoire (et justifiant souvent son financement via des contrats "Génome "), d'autre part un sujet personnel plus ciblé rattaché à ce thème mais susceptible de s'avérer intéressant et rentable en termes de résultats publiables. C'est une tactique qui peut être payante si ces sujets secondaires sont astucieusement choisis de façon à profiter au maximum des clones, des données acquises dans le cadre du programme principal ainsi que des méthodes mises en place pour son exécution. Mais elle porte en elle le risque d'une dérive, le danger qu'imperceptiblement le projet latéral devienne la priorité du chercheur et que l'autre activité soit délaissée : dérive bien compréhensible... L'histoire récente du tri de chromosomes dans le laboratoire de Lawrence Livermore, en Californie, en fournit un exemple. Cette équipe, avec celle de Los Alamos au Nouveau Mexique, a été parmi les premières à maîtriser la préparation de banques spécifiques à partir de chromosomes ainsi triés. Les deux centres se sont alors partagé les chromosomes humains afin d'établir, au service de la communauté, un jeu complet de librairies spécifiques pour chaque chromosome, tout en continuant à avoir une activité de recherche concernant l'amélioration des méthodes de tri et leur emploi pour étudier le polymorphisme de taille des chromosomes. Le parti choisi à Lawrence Livermore a été d'affecter une machine au tri de routine (celui qui sert à la construction de banques), une autre au tri de recherche. Bien que l'ensemble soit sous la responsabilité des mêmes scientifiques, il s'est produit progressivement un glissement qui a abouti à ce que l'essentiel de l'attention se porte sur le tri de recherche et la machine correspondante. La qualité des banques produites (et distribuées) par Lawrence Livermore n'a pas tardé à s'en ressentir; il s'est trouvé en particulier que plusieurs préparations de chromosomes ont été contaminées par des bactéries du type Pseudomonas et que les banques résultantes contenaient quelques pour cent de clones provenant de l'ADN de cette bactérie. Cette contamination était catastrophique pour certains utilisateurs : ces banques ont en effet souvent été employées pour construire des sousbanques d'îlots $\mathrm{CpG}$, pour lesquelles on sélectionne par divers artifices les clones dont l'ADN contient des séquences riches en $\mathrm{CpG}$, qui correspondent aux "îlots HTF ", associés en général à des gènes. Or l'ADN de Pseudomonas contient une forte proportion de ce dinucléotide... de sorte que les banques d' "îlots HTF " obtenues à partir de ces librairies contenaient surtout des clones de Pseudomonas... 
On voit que la dissociation entre les deux aspects du travail est très dangereuse : la solution a consisté à rénover et améliorer les machines et surtout à établir un planning selon lequel la même machine est utilisée alternativement une semaine pour le tri de routine et une pour la recherche : ainsi s'assure-t-on d'un intérêt soutenu des chercheurs pour le parfait état du matériel qui sert pour le tri de routine. Mais le meilleur exemple d'intégration entre recherche ciblée et travail systématique, je l'ai rencontré dans le laboratoire de David Ward, pape des sondes non radioactives et apôtre de l'hybridation in situ. On connaît ses études sur la mise au point et la diffusion de l'hybridation in situ avec des sondes non radioactives ; elles ont transformé une technique longue et délicate, valable seulement entre les mains de quelques spécialistes et relativement imprécise du fait de la taille des grains d'argent qui signalent la sonde... en une méthode fiable et rapide, transférable dans de nombreux laboratoires et avec laquelle un post-doc peut localiser une centaine de sondes en deux ou trois mois (à condition que ces sondes soient des cosmides, ce qui tend à devenir le cas général). Son laboratoire place ainsi plus de mille sondes par an : des cosmides provenant de banques spécifiques d'un chromosome, des linking clones employés pour la cartographie en champs pulsés..., dans le cadre d'une multitude de projets. On imagine une armée de techniciens rivés à leurs microscopes et faisant de l'in situ à longueur de journée : il n'en est rien, et l'organisation du laboratoire est très instructive. En fait chaque post-doc (il y en a une douzaine) a son propre sujet, centré sur le clonage du gène d'une maladie pour laquelle on a déjà une localisation approximative (liaison génétique, ou translocation). Le travail commence alors par l'étude de quelques dizaines de sondes (en général des cosmides) provenant de la région ou, à défaut, d'une librairie spécifique du chromosome en question ; elles sont localisées par hybridation in situ sur métaphases et les mieux placées servent alors à une cartographie fine sur les noyaux en interphase. On res- treint ainsi la zone où peut se trouver le gène à quelques centaines de kilobases, dans lesquelles l'on dispose maintenant de plusieurs sondes ; l'approche est d'ailleurs considérablement accélérée s'il existe une translocation dans la région, ce que l'on recherche aussi par hybridation in situ sur des noyaux en interphase. Le post-doc est alors prêt à passer aux YAC et aux ADNc pour l'étape suivante de sa recherche. Avec ce système d'une redoutable efficacité, chacun fait quelques mois d'in situ à haute dose, plaçant une centaine de sondes dans le cadre de son projet. Les critères de validité sont clairement définis et un dispositif de vérification a été élaboré pour confirmer les localisations avant de les inscrire dans la base de données du laboratoire qui "produit " ainsi de très nombreuses localisations alors qu'il ne comprend qu'un seul technicien. Bref, une manière de travailler originale, très efficace, semblant résoudre la contradiction qui parasite beaucoup de projets Génome. Cette solution n'est possible que parce que les méthodes d'hybridation in situ pratiquées dans ce laboratoire sont en avance sur ce qui se fait presque partout ailleurs : cet avantage technique joue un rôle déterminant. Si les post docs de Dave Ward mettaient une ou deux années pour localiser leurs cent sondes, ou si tout le monde pouvait localiser cent cosmides en trois mois, cette organisation astucieuse ne tiendrait plus. L'élégante solution que nous venons d'explorer n'est donc malheureusement pas généralisable. L'automatisation, une porte de
sortie?

L'automatisation ne pourrait-elle résoudre cette contradiction ? A l'ère des microprocesseurs et de la robotique, quoi de plus simple en apparence que de remplacer le personnel souvent surqualifié qui effectue les tâches manuelles de la recherche génomique par des automates précis, rigoureux, fiables et surtout infatigables ? Ces systèmes encore très peu répandus chez nous, je pensais les rencontrer en grand nombre au cours de mon enquête. Aux États-Unis notamment, berceau du " Programme Génome ", j'espérais beaucoup des centres du DOE. Cet organisme (Department of Energy), qui avait pris l'initiative du programme au milieu des années 1980, a une tradition technologique très marquée, liée tout naturellement à son implication dans des programmes high-tech comme la bombe atomique et, plus récemment, la "guerre des étoiles ": on pouvait donc s'attendre à y voir un développement massif des approches instrumentales de la biologie. Ce n'est, en fait, pas vraiment le cas. Il y a certes dans ces laboratoires (Lawrence Livermore et Lawrence Berkeley en Californie, Los Alamos au NouveauMexique) plus de technologie qu'ailleurs, mais le travail à la main continue à prédominer. On y rencontre tout juste un ou deux robots Beckman "Biomek ", ce robot de pipetage qui commence à être employé pour certaines opérations de Biologie moléculaire : duplication de cultures stockées en plaques à microtitration, confection de filtres à partir de ces mêmes microplaques, réactions de séquençage... Les équipes de robotique (car il y en a) travaillent sur la construction, à partir d'éléments du commerce, de robots du même type mais plus rapides ou plus précis, ou sur la mise au point de systèmes pouvant reconnaître et prélever des colonies sur une boîte de Petri, ou encore sur des machines à PCR capables d'accepter un très grand nombre d'échantillons ; mais le pratique quotidienne des laboratoires reste pour l'essentiel celle du travail manuel. Relative déception donc, décidément la robotique a bien du mal à pénétrer dans les laboratoires de biologie, même dans ceux qui devraient en principe y être le plus perméables... Quelles sont les raisons d'un tel retard? Tout d'abord le conservatisme du milieu de la recherche biologique fondamentale, où l'on hésite souvent à investir le temps indispensable à la mise au point d'une nouvelle procédure (qui serait pourtant rentable dans le long terme). Une autre raison est le manque de formation en technologie et en physique de base de la plupart des biologistes, qui rend le dialogue avec un ingénieur en robotique bien difficile... Les équipes 
du DOE ont pourtant une culture technique très supérieure à la moyenne ; mais même dans ces centres le fossé entre biologistes et roboticiens, comme entre biologistes et informaticiens, est profond : les cultures diffèrent, la perception des impératifs aussi. Les spécialistes veulent réaliser un programme ou une machine élégants, parfaits à leur point de vue ; le biologiste, lui, cherche plutôt la rapidité de mise à disposition d'un outil même un peu approximatif, et la facilité d'emploi. D'autres raisons, plus fondamentales, expliquent ce retard. La principale sans doute est que les techniques du génie génétique sont encore en évolution rapide. Compte tenu des délais qu'implique la mise au point puis l'éventuelle commercialisation d'un automate spécialisé, le risque que celui-ci soit obsolète au moment de son introduction est réel. La montée en puissance des méthodes utilisant l'amplification enzymatique (PCR) en est un bon exemple : l'amplification court-circuite toute une série d'étapes et change complètement l'ordre de grandeur de la sensibilité nécessaire dans de nombreuses applications puisque la région à examiner peut maintenant être amplifiée spécifiquement un million de fois au préalable. Du coup le marché potentiel d'une machine à réaliser les Southern blots se trouve considérablement rétréci puisque la PCR permet souvent d'éviter le recours à cette technique. Robotiser, c'est ainsi faire un pari risqué, surtout pour un industriel ; c'est courir le danger d'aboutir à un appareil dépassé par l'évolution des techniques ou simplement trop cher pour les laboratoires de biologie où une machine de $500 \mathrm{KF}$ est encore considéréc comme du gros matériel (l'échelle n'est pas la même que pour nos collègues physiciens...).

L'automatisation ne peut donc apporter qu'une solution très partielle à notre dilemme - ce qui ne veut pas dire qu'il faille la négliger, ni qu'elle soit employéc aujourd'hui au maximum de ses possibilités... Mais sa mise en œuvre s'avère délicate, en raison d'obstacles techniques, financiers et psychologiques. On peut alors penser à contourner l'obstacle en opérant une division du travail : la part systématique et bien codifiée serait assurée par quelques "Centres de Service " organisés de manière industrielle, équipés de machines et bien dotés en personnel technique; les laboratoires auraient recours à ces centres pour les phases correspondantes de leur travail, gardant la maîtrise de leurs projets et la responsabilité de la conception et de l'exécution des parties les plus nobles mais aussi les plus délicates de leurs programmes. Il s'agirait en somme de constituer des sortes de services communs pour le Génome.

Services communs : efficacité non garantie

Les services communs ont mauvaise presse en France, et il faut reconnaître que beaucoup d'entre eux, édifiés à grands frais et dotés d'un abondant personnel, se sont révélés remarquablement peu performants. On se souvient sans doute du Centre CNRS d'élevage d'animaux de laboratoire à Orléans, qui a pendant des années fourni à nos laboratoires des souris dont le prix de revient réel (salaires et amortissements compris) était très supérieur à celui que font payer des fournisseurs commerciaux qui ne sont pourtant pas, que l'on sache, des philanthropes. On pourrait facilement multiplier les exemples. D'autres services communs fonctionnent, eux, relativement bien, mais à l'avantage quasiment exclusif d'un laboratoire : ils lui sont pratiquement intégrés (ce qui est sans doute la raison de leur relative efficacité) et n'ont de commun que le nom : ils constituent en fait une façon déguisée d'augmenter le potentiel de l'équipe en question. $\mathrm{Au}$ moins peut-on apprécier que dans ce cas les deniers publics ne soient pas gaspillés, même s'ils n'ont pas exactement l'usage prévu. Mais globalement la situation est loin d'être satisfaisante, et la tendance depuis plusieurs années est à la fermeture de ces services, les tâches correspondantes étant prises en charge soit par le secteur privé (une bonne solution lorsqu'on peut faire jouer la concurrence) soit par des structures inté- grées aux laboratoires et bénéficiant, on l'espère, de leur dynamisme. L'échec généralement reconnu des services communs en France s'explique aussi par certaines de nos tares nationales : notre répugnance à mettre en question les structures une fois qu'elles ont été créées, une culture économique très lacunaire dans le secteur public, et l'incontournable problème du statut du personnel... car naturellement tout le monde dans ces lieux est fonctionnaire INSERM ou CNRS, ce qui empêche le recrutement de certains hommes clefs au niveau de qualification voulu (en raison de la modicité des salaires), gêne toute adaptation en profondeur et rend très malaisée la remise en cause de la structure.

Mais ces paramètres ne sont pas les seuls, et même dans des systèmes très différents de nos organismes publics, les "Centres de Ressources" et autres services communs se heurtent à quelques difficultés. Par exemple, l'évaluation quantitative et qualitative de la demande des futurs utilisateurs n'est pas évidente, et il ne faut pas faire trop confiance aux prévisions qu'ils expriment et qui se révèlent souvent dramatiquement fausses. Les usagers savent aussi se montrer parfaitement déraisonnables, surtout si cela ne leur coûte rien : David Schlessiger, dont le laboratoire effectua généreusement (et gratuitement) une série de criblages par hybridation de sa banque YAC en 1988/89 pour des laboratoires extérieurs en parle encore avec amertume. Il ne compte plus les cas où les sondes reçues s'avéraient inutilisables, et se souvient de ce laboratoire rappelant un an après avoir reçu les précieux clones YAC tirés (au prix de beaucoup d'efforts) de la banque, pour les réclamer de nouveau : ils n'en avaient rien fait dans l'intervalle et les avaient perdus, ou laissé se dessécher dans leur réfrigérateur... Si le groupe de Saint-Louis s'est rapidement converti au criblage par PCR c'est sans doute parce que cette méthode est plus fiable (encore que Hans Lehrach (ICRF, Londres) soit d'un autre avis), mais surtout parce qu'elle exige du laboratoire demandeur un certain effort : séquencer sa sonde, définir et synthétiser des oli- 
gonucléotides, vérifier qu'ils donnent une bande nette et unique par amplification sur de l'ADN génomique... Si le demandeur a fait ce travail, on peut supposer qu'il a réellement besoin du clone YAC qui sera éventuellement obtenu, ce qui est rassurant pour ceux qui se livreront au lourd travail du criblage. Un autre moyen de filtrer les demandes frivoles est de faire payer à leurs auteurs une somme raisonnable, mesure qui est souvent plus utile par son effet dissuasif que par la rentrée financière qu'elle occasionne. Les centres de service ont aussi du mal à obtenir des utilisateurs des informations, un feedback comme on dit en franglais, sur le devenir des objets qui leur ont été fournis : qualité des librairies, caractéristiques des clones... ces informations reviennent au "fournisseur", auquel elles seraient pourtant précieuses, ne serait-ce que pour mieux connaître la qualité de ses produits. De ce point de vue le système des librairies de référence de Hans Lehrach a l'avantage d'imposer un minimum de retour d'informations puisque c'est à partir des coordonnées sur le filtre du ou des clones positifs en hybridation que pourront être fournis ces derniers. En tout état de cause, il est important pour un centre de service d'avoir un cahier des charges précis et d'indiquer très nettement quels services il peut rendre, et dans quelles conditions; sinon il devient vite parasité par des circuits parallèles. Les modes d'emploi du HGMP Resource Centre, largement diffusés dans le petit journal G-Nome News représentent sans nul doute un exemple à suivre. Le centre de service doit aussi avoir une politique claire au niveau des signatures d'articles : dans quels cas se comporte-t-il en prestataire de services, quand y a-t-il en revanche, collaboration scientifique avec en corollaire participation aux articles ? Là aussi les règles doivent être explicites.

La situation aux États-Unis mérite aussi quelques développements. Il n'existe pas à ma connaissance dans ce pays de centres de service au sens strict, peut-être parce qu'ils sont moins nécessaires qu'en Europe en raison de la relative abondance des financements. En fait chaque Centre d'études sur le Génome un peu important s'est rendu autonome en obtenant, par exemple, une copie de la banque YAC de Saint-Louis et même d'une ou deux banques européennes. Il n'y a donc pas de système de criblage à façon (par PCR) comme celui offert en GrandeBretagne ou, en France, par le $\mathrm{CEPH}$, ce qui handicape les laboratoires qui n'ont pas de relations avec un Genome center. Les contrats Génome prévoient explicitement le soutien de services, souvent appelés cores ; ils font même l'objet au NIH d'un contrat spécial distinct des contrats de recherche. Mais ces core services m'ont paru en général très centrés sur le service... local, comme par exemple dans le département de génétique du Baylor College (Tom Caskey, Houston, Texas) où ils semblent surtout soutenir les (excellentes) équipes de génétique humaine du Département. On trouve à leur tête des chercheurs qui ont fait le choix d'un travail plus routinier, moins "glorieux" que les autres équipes, mais avec par contre une situation relativement stable et un répit dans l'épuisante chasse au contrat. Rappelons en effet que ce département, comme beaucoup aux Etats-Unis, offre aux équipes de recherche le gîte, mais pas le couvert, qu'elles dépendent de leurs contrats pour la quasitotalité de leurs fonds de recherche, pour leur personnel et même pour la majeure partie du salaire de leur responsable...

\section{Comment attirer des seniors}

C'est une des questions les plus difficiles à résoudre pour les centres de ressource. Les responsables du HGMP resource Centre de Harrow, dans la banlieue de Londres, le centre de service principal du programme Génome britannique, la considèrent comme leur problème principal. Le Resource Centre, déjà mentionné à propos du programme Génome britannique [1], est un " vrai " centre de service : entendez qu'il se préoccupe essentiellement d'apporter à ses utilisateurs britanni- ques (et européens, on ne sait pas assez qu'il est ouvert à tout laboratoire de notre continent, ou du moins de la CEE) les services qu'ils demandent. Cela comprend le criblage de banques YAC (celles de Saint-Louis et de Rakesh Anand), la fourniture de sondes et d'amorces PCR, bientôt la localisation de sondes chez la souris dans le cadre du backcross européen, des banques d'ADNc, l'accès aux bases de données et une aide à l'emploi de systèmes informatiques... Structure de taille modérée, créée de façon très pragmatique et avec des moyens raisonnables, elle souffre de ne pouvoir attirer quelques biologistes de haut niveau. Son personnel, formé de jeunes au profil michercheur mi-ingénieur avec un niveau proche du $\mathrm{PhD}$, a une qualification suffisante pour effectuer les travaux courants ; mais quand quelque chose ne va pas, quand une méthode ne "marche" plus sans raison apparente ou que la mise en place d'une nouvelle technique ne réussit pas, l'absence de chercheurs chevronnés capables de faire le troubleshooting nécessaire se fait cruellement sentir. Jusqu'à maintenant les efforts faits pour en attirer ont échoué à cause du manque d'intérêt de ce travail de routine pour un bon chercheur ; l'environnement peu attrayant (Harrow est une banlieue très lointaine, et il y a peu de recherche sur place : le Resource Centre est installé dans les locaux d'une unité du MRC fermée pour qualité scientifique insuffisante) joue sans doute aussi son rôle.

La situation actuelle du "Généthon " créé à grands frais par l'AFM à Évry avec l'expertise de CEPH montre comment les choses peuvent évoluer lorsqu'on tente de résoudre cette question en injectant de la recherche dans une structure de service. Lors de sa création en 1990 le Généthon avait en effet été présenté comme une structure où seraient rassemblés équipement lourd et personnel technique dont bénéficieraient les équipes de recherche françaises à certaines phases critiques de leurs travaux. Aux étapes particulièrement laborieuses impliquant la réalisation de centaines de Southern blots, un séquençage massif ou encore l'alignement de nom- 
breux clones, ces équipes pourraient venir utiliser pendant une période limitée les équipements et le savoirfaire rassemblés à Évry avant de repartir poursuivre leurs travaux " chez elles". Le démarrage de cet ensemble a, en fait, vite fait apparaître la pénurie de cadres; et pour en attirer, au moins à temps partiel, dans ce bâtiment très bien équipé mais un peu perdu dans une banlieue lointaine, la décision a été prise d'y implanter des projets de recherche. Plusieurs d'entre eux sont maintenant opérationnels ; le plus avancé est celui que dirige Jean Weissenbach et qui vise à définir un très grand nombre de microsatellites hautement polymorphiques af in d'apporter une contribution décisive à l'affinement de la carte génétique humaine. Parmi les autres thèmes on doit citer celui de Daniel Cohen sur l'" extraction " de banques YAC spécifiques à partir de la banque générale du CEPH et la construction de contigs le long du chromosome 21, et bien sûr le projet " Genexpress " de Charles Auffray (en principe cofinancé par le CNRS) qui a pour objectif un séquençage massif et partiel de clones d'ADNc selon un schéma assez analogue à celui popularisé par Craig Venter. Trois études (il y en a d'autres) innovantes, et qui ont de bonnes chances de succès; mais ce sont des sujets de recherche, même s'ils doivent à terme déboucher sur des réactifs d'intérêt général, et l'aspect " service " primordial dans le schéma initial est un peu passé au second plan. Au lieu d'être une structure originale qui allait permetre à de nombreux laboratoires d'accéder, au moment venu, aux techniques lourdes et ainsi irriguer l'ensemble des travaux sur le génome en France, le Généthon est devenu une sorte de deuxième $\mathrm{CEPH}$, effectuant avec de très gros moyens (chacun des thèmes cités cidessus emploie une vingtaine de techniciens, sans parler des machines) une recherche de pointe. Il convient de tempérer cette appréciation en rappelant que Généthon a aussi des activités ouvertes sur l'extérieur, banques de cellules, séquence à façon (dans quelques cas), et confection de Southern blots pour différents projets; mais même l'atelier des automates à blots tourne à plus de $50 \%$ pour les $\mathrm{m} / \mathrm{s} n^{\circ} 5$, vol. 8, mai 92 besoins " internes " $(\mathrm{CEPH}$ et recherches menées sur place).

\section{Rapport qualité/prix : la bouteille à l'encre}

Tout ce travail coûte cher, très cher. Les appareils valant moins de 0,5 ou $1 \mathrm{MF}$ deviennent rares ; l'informatique, malgré la baisse constante des prix, absorbe des sommes élevées; et la base de calcul du financement pour certaines structures proposées s'approche du MF par personne, somme qui inclut, il faut le dire, la plupart des salaires. On atteint là des sommets qui n'ont plus rien à voir avec les dépenses d'un laboratoire classique, estimées, pour des unités bien financées et faisant beaucoup de biologie moléculaire et de culture de cellules, à deux ou trois fois moins. Certes une activité de service, fortement automatisée et donc consommant de grandes quantités de réactifs, ouverte sur l'extérieur et " exportant " des objets onéreux à produire, une telle activité doit logiquement exiger un soutien plus lourd qu'une recherche académique classique. Mais l'évaluation précise devient très délicate, et n'est pas la moindre difficulté de ce secteur. On ne dispose pas ici de l'étalon commode du fournisseur de souris commercial qui permettait d'évaluer facilement la viabilité (ou plutôt la non-viabilité) du CSEAL ; encore qu'il commence à y avoir des firmes qui font de la séquence à façon, et que si l'entreprise envisagée par le milliardaire Fréderick Bourke [2] se réalise effectivement, elle pourrait fournir une référence bien utile. Mon impression, fondée sur la visite de nombreux centres et la communication de leurs budgets, est que le rapport qualité/prix est extraordinairement variable : selon la stratégie choisie, la pertinence des choix faits, la rigueur de leur mise en œuvre, la qualité de la gestion du centre... je ne serais pas étonné qu'il y ait des écarts de un à dix dans le prix de revient, par exemple, de la mégabase de contig de YAC. Evaluation malaisée, mais pourtant primordiale, car avec la nature intrinsèquement expansionniste des programmes
Génome il est impératif de dépenser au mieux les sommes importantes qui leur sont allouées.

\section{Changement d'échelle, changement de cultures?}

Beaucoup des questions évoquées cidessus découlent des dimensions du programme Génome et de son caractère systématique. C'est en effet la première fois qu'est entrepris en Biologie un effort aussi structuré : la " guerre contre le cancer " lancée du temps de Richard Nixon, ou les recherches sur le SIDA aujourd'hui ont une ampleur comparable mais regroupent une multiplicité de travaux très divers, dont la plupart pris isolément peuvent s'inscrire dans un cadre "académique " classique. Au contraire, les tâches définies par ce projet sont bien codifiées : affiner la carte génétique jusqu'à une résolution de deux à quatre centimorgans, établir des cartes physiques de chromosomes entiers, séquencer de grandes régions d'ADN. Leur accomplissement dans de bonnes conditions impose la mise en place d'une organisation de type semi-industriel, assurant un suivi précis de l'avancement des projets et leur contrôle de qualité, ainsi qu'une gestion professionnelle sur le plan financier... toutes choses auxquelles l'on n'est pas trop habitué dans les laboratoires de recherche fondamentale. Pour autant, le fonctionnement doit rester extrêmement souple et se prêter à la remise en cause des stratégies expérimentales (nous avons lourdement insisté sur le caractère évolutif des techniques), remise en cause qui doit s'étendre aux structures et aux hommes qui les animent. C'est un peu la quadrature du cercle... d'autant qu'il ne faut pas dans tout cela oublier la motivation de ceux qui exécutent ce travail. A cet égard, il faudra bien arriver à une meilleure reconnaissance des travaux systématiques, à attacher par exemple une certaine valeur à la production d'informations qui sont inscrites dans des bases de données, sans passer par une publication au sens habituel du terme. Une petite révolution culturelle, en quelque sorte...
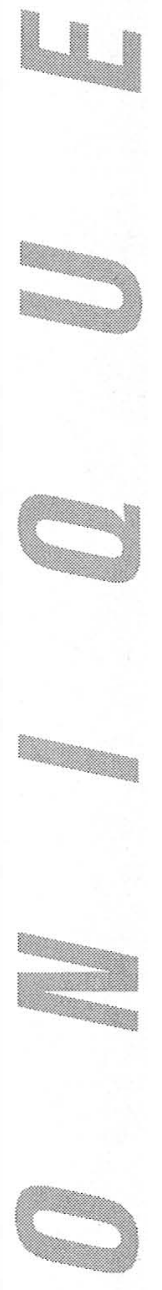

\section{(1) .

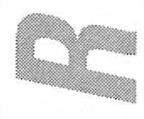

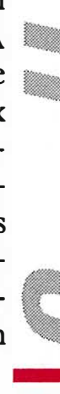


Les programmes Génome constituent, on le voit, une sorte de banc d'essai d'une biologie différente, plus massive, plus instrumentale, plus industrielle ; et la sociologie de cette nouvelle façon de faire de la recherche n'est pas sans intérêt. Les questions qui sont posées nous amènent en effet à remettre en cause certains comportements hérités d'une ère individualiste et artisanale dont le champ est sans doute en train de se rétrécir

Bertrand Jordan

Directeur de recherche au Cnrs, responsable $d u$ groupe de génétique moléculaire humaine. CIML, Inserm/Cnrs, case 906, 13288 Marseille Cedex 9, France.

\section{RÉFÉRENCES}

1. Jordan B, Grande-Bretagne : un programme Génome à dimension humaine. médecine/sciences $1992 ; 8$ : 163-6.

2. Anderson $\mathrm{C}$, Aldhous $\mathrm{P}$. Genome project faces commercialization test. Nature $1992 ; 355: 483-4$.

\section{TIRÉS A PART}

B. Jordan. 